\title{
Estimation and Statistical Bounds for Three-Dimensional Polar Shapes in Diffuse Optical Tomography
}

\author{
Gregory Boverman*, Member, IEEE, Eric L. Miller, Senior Member, IEEE, Dana H. Brooks, Senior Member, IEEE, \\ David Isaacson, Qianqian Fang, Member, IEEE, and David A. Boas
}

\begin{abstract}
Voxel-based reconstructions in diffuse optical tomography (DOT) using a quadratic regularization functional tend to produce very smooth images due to the attenuation of high spatial frequencies. This then causes difficulty in estimating the spatial extent and contrast of anomalous regions such as tumors. Given an assumption that the target image is piecewise constant, we can employ a parametric model to estimate the boundaries and contrast of an inhomogeneity directly. In this paper, we describe a method to directly reconstruct such a shape boundary from diffuse optical measurements. We parameterized the object boundary using a spherical harmonic basis, and derived a method to compute sensitivities of measurements with respect to shape parameters. We introduced a centroid constraint to ensure uniqueness of the combined shape/center parameter estimate, and a projected Newton method was utilized to optimize the object center position and shape parameters simultaneously. Using the shape Jacobian, we also computed the Cramér-Rao lower bound on the theoretical estimator accuracy given a particular measurement configuration, object shape, and level of measurement noise. Knowledge of the shape sensitivity matrix and of the measurement noise variance allows us to visualize the shape uncertainty region in three dimensions, giving a confidence region for our shape estimate. We have implemented our shape reconstruction method, using a finitedifference-based forward model to compute the forward and adjoint fields. Reconstruction results are shown for a number of simulated target shapes, and we investigate the problem of model order selection using realistic levels of measurement noise. Assuming a signal-to-noise ratio in the amplitude measurements of $40 \mathrm{~dB}$ and a standard deviation in the phase measurements of $0.1^{\circ}$, we are able to estimate an object represented with an eighth-order spherical harmonic model having an absorption contrast of $0.15 \mathrm{~cm}^{-1}$ and a volume of $4.82 \mathrm{~cm}^{3}$ with errors of less than $10 \%$ in object volume and absorption contrast. We also investigate the robustness of our
\end{abstract}

Manuscript received May 3, 2007; revised August 3, 2007. The work of G. Boverman, D. H. Brooks, D. Isaacson, and E. L. Miller was supported by the Center for Subsurface Sensing and Imaging System (CenSSIS) under the Engineering Research Centers Program of the National Science Foundation under Award EEC-9986821. The work of E. L. Miller was also supported by the National Science Foundation under Award 0208548 and Award 0139968. The work of Q. Fang and D. A. Boas was supported by the National Institutes of Health. Asterisk indicates corresponding author.

${ }^{*} \mathrm{G}$. Boverman is with the Biomedical Engineering Department, Rensselaer Polytechnic Institute, 7046 Jonsson Engineering Center, Troy, NY 12180 USA (e-mail: boverg@rpi.edu).

E. L. Miller is with the Department of Electrical and Computer Engineering, Tufts University, Medford, MA 02155 USA.

D. H. Brooks is with the Department of Electrical and Computer Engineering, Northeastern University, Boston, MA 02115 USA.

D. Isaacson is with the Department of Mathematical Sciences, Rensselaer Polytechnic Institute, Troy, NY 12180 USA.

Q. Fang and D. A. Boas are with the Martinos Center for Biomedical Imaging, Massachusetts General Hospital, Charlestown, MA 02129 USA.

Color versions of one or more of the figures in this paper are available online at http://ieeexplore.ieee.org.

Digital Object Identifier 10.1109/TMI.2007.911492 shape-based reconstruction approach to a violation of the assumption that the medium is purely piecewise constant.

Index Terms-optical tomography, image reconstruction, inverse problems, shape-based imaging.

\section{INTRODUCTION}

D IFFUSE optical tomography (DOT) is an emerging imaging modality which is beginning to show great promise, primarily due to its ability to monitor the body's hemodynamic properties in vivo using near-infrared light. Among DOT's advantages over conventional imaging modalities in widespread use are its relatively low cost and the employment of safe, nonionizing radiation. However, image reconstruction for DOT is difficult and relatively computationally expensive, primarily due to the ill-posedness and nonlinearity of the inverse problem. The ill-posedness is caused by the multiple scattering that photons experience in their path from source to detector, with the result that a typical measurement is affected by the absorption and scattering over a large volume of the imaging geometry. If it is assumed that the forward problem can be linearized, it is possible to compute the analytical solution to the regularized inverse problem for a number of specialized imaging geometries [1], but it is not currently possibly to do so for more complex geometries such as the breast. In the case of the fully nonlinear inverse problem, which we address in this paper, analytical methods may be applicable for the solution of the inverse problem [2], but again only for specific imaging geometries, and they require the assumption that the scattering series can be truncated to a particular order. Thus, for realistic imaging geometries, nonlinear optimization methods must be used. In this paper, we will focus on the application of DOT for breast tumor detection and characterization. Recent experimental work has imaged tumors with DOT and has suggested that tumors may differ sharply from normal tissue in their blood volume, oxygenation, and, perhaps in their scattering properties [3]-[5].

The most common approach to the DOT inverse problem in the literature is to estimate optical parameter distributions which minimize a cost functional [6], [7]. This cost functional typically includes a term which represents the mismatch between the actual measurements and the measurements which would have resulted from the estimated absorption and scattering distribution, generally computed by means of a forward solver. An additional term is included for the purpose of regularization, penalizing 
some function of the estimates themselves, such as their norms, or the norm of their gradients. The estimation problem, having been reduced to a nonlinear regression problem, can be solved by means of gradient [8] or Newton-based [7], [9] approaches, among others. The functional gradient of the cost function with respect to absorption perturbation can be computed efficiently [10]. The resulting solution is highly dependent on the choice of a regularization parameter, the selection of which has been quite exhaustively discussed in the inverse problems literature [11], [12].

However, using standard approaches, recovery of edges in the DOT inverse problem is extremely difficult because the propagation of diffuse light highly attenuates absorption distributions with high spatial frequency. Thus, inversion methods must make use of regularization to prevent the resulting reconstructions from being overwhelmed by noise. As a result, the preponderance of DOT image reconstructions reported have little discernible shape [3], [13]. One approach taken in the literature on image reconstruction in order to deal with this problem is to incorporate total-variation minimization [14] or edge-preserving regularization [15] into the image reconstruction. This approach has also been useful in the related applications of image restoration [16], [17] and image segmentation [18], [19].

In this paper, we will show that, given the assumption that the medium is piecewise constant, we can reconstruct the shape, optical absorption contrast, and location of an anomaly. We recover boundary information by explicitly postulating the existence of a compactly supported absorbing anomaly, estimating the shape of this inclusion using a nonlinear optimization method. While it is not exactly true that the body, or the breast in particular, is a piecewise-constant medium, it is likely to be composed of distinct regions (i.e., adipose tissue, glandular tissue, tumor tissue), with clear boundaries, and where the mean of the optical properties within each region differs significantly from that of the other regions [20]. In this paper, we consider a simpler problem in which we are concerned only with the estimation of a single inclusion embedded in an otherwise homogeneous medium. We demonstrate the properties of this approach on simulated data with varying levels of measurement noise.

Recent shape-based work has made use of level-set functions [21], active contours [22], and various boundary parametrizations. In two dimensions, Fourier descriptors [23], [24] and B-spline models [25] have been employed to parameterize the boundary. For DOT, the first work in this area examined the estimation of the location, contrast and orientation of ellipsoids [26]. More recently, spherical harmonic shape parametrizations have been used in conjunction with the boundary element method (BEM) [27], [28]. In our work, we use spherical harmonics to model fairly intricate 3-D polar shapes, as described by $\mathrm{Li}$ [19] (a polar shape is one that can be modeled as a single-valued function in spherical coordinates), with respect to some center position. We show that we can efficiently solve the inverse problem, using shape derivative theory and the adjoint method to compute the derivatives of our measurements as we vary our shape parameters. We also introduce a centroid constraint to allow simultaneous estimation of object position and shape.
An advantage of our approach is that the number of terms used to represent an object in the inversion plays the role of a regularization parameter. Given noisy measurements, it is not possible to reconstruct the shape of an object exactly from external diffuse optical measurements of intensity and phase. A parametric representation allows us to vary the complexity of our model depending on the accuracy of our measurements. In our simulation studies, we consider the model-order selection problem is some detail. In addition, our shape reconstruction approach extends to the case of multiple objects with different contrasts relatively easily, although for such an approach one must either know the number of objects a priori or embed in the processing a method for determining this quantity.

We will make use of the domain, or Fréchet derivative of the scattered field with respect to infinitesimal perturbations of the object boundary, as described by Sokolowski and Zolesio [29]. The domain derivative approach was applied to the electromagnetic inverse problem for the case of a perfectly conducting obstacle by Kirsch [30]. Hettlich [31] extended Kirsch's variational approach to compute the Fréchet derivative of the scattered field with respect to boundary perturbations for a number of object boundary conditions. The domain derivative can be computed as the solution of a forward problem with particular boundary conditions defined on the object boundary. Potthast [32] gave an alternative derivation for the scattered field domain derivative, assuming a $C^{2}$ continuously differentiable boundary. Hohage and Schormann [33] extended this method to compute the Fréchet derivative of the scattered field for perturbation of the boundary of a penetrable obstacle.

In addition, knowledge of the shape and center Jacobian matrices allows us to compute the constrained Cramér-Rao lower bound on shape estimation accuracy and to visualize this bound. This bound tells us the best reliability that is possible in our shape reconstruction, given a particular measurement geometry and measurement noise level. This knowledge, apart from giving us a shape "confidence interval," can help us in our design of instruments and source/detector layouts, if we have prior knowledge that a particular shape reconstruction reliability is desired. In contrast to previous work on shape estimation bounds in image reconstruction problems [23], [25], [34], we derive and implement these bounds specifically for the DOT inverse problem, and for the very challenging problem of fully 3-D nonlinear shape estimation. We visualize these bounds using a method introduced by Kirsch [30], extended to the case of 3-D polar shapes. As we have introduced a centroid constraint to ensure uniqueness of our object shape/center parametric representation, we make use of the constrained Cramér-Rao lower bound [35] to give us performance bounds on simultaneous estimation of object shape and center.

In our experimental work, we show in simulations that fairly complex objects can be estimated with a high degree of accuracy using DOT data and a reasonable level of noise. We also show shape reconstruction performance where there is a trade-off between model complexity and estimation accuracy, since overly complex models cannot be accurately estimated using a limited set of noisy measurements. In addition, we consider the case where the medium is not purely piecewise-constant, using a simulation in which variation is introduced into the absorption in 
both the object and in the background. We show that our algorithm is robust to this form of model mismatch but that the degree of error introduced is dependent on the variance of the perturbations in the background. We examine the question of model order selection, showing that a sufficient level of model complexity is necessary in order to estimate an absorber's size and contrast accurately, but that the model complexity need not be identical to the object's true complexity in order to achieve reasonably accurate estimates. Finally, we look to the question of shape reconstruction lower bounds, visualizing the shape bounds derived from the parametric Cramér-Rao bounds, using both the unconstrained bound and the constrained bound, where a centroid constraint has been introduced.

\section{SHAPE PARAMETERIZATION}

In order to reduce the complexity of the 3-D shape estimation problem, we make the assumption that we are reconstructing polar shapes, which can be expressed as single-valued functions in spherical coordinates, with respect to some center location. In this context, spherical harmonics are a very useful choice for shape parametrization, as they define an orthonormal basis on the unit sphere. Since spherical harmonics are complex functions, we make use of their real and imaginary parts, the Tesseral harmonics, in order to ensure that our shape estimate is strictly real. The Tesseral harmonics of order 4 and below are shown in Fig. 1.

The spherical harmonics are defined as follows [36]:

$$
Y_{l}^{m}(\theta, \phi)=(-1)^{m} \sqrt{\frac{2 l+1}{4 \pi} \frac{(l-m) !}{(l+m) !}} P_{l}^{m}(\cos \theta) e^{i m \phi}
$$

where $\theta \in[0, \pi)$ and $\phi \in[0,2 \pi)$ and $P_{l}^{m}(x)$ is the associated Legendre function of order $l$ where $-l \leq m \leq l$. Therefore, the radius with respect to a given center position can be represented as follows [19]:

$$
R(\theta, \phi, \mathbf{a})=\sqrt{\sum_{l=0}^{N} \sum_{m=0}^{l} a_{l}^{m} U_{l}^{m}(\theta, \phi)+b_{l}^{m} V_{l}^{m}(\theta, \phi)+\epsilon}
$$

where $U_{l}^{m}=\mathcal{R}\left\{Y_{l}^{m}(\theta, \phi)\right\}$ and $V_{l}^{m}=\mathcal{I}\left\{Y_{l}^{m}(\theta, \phi\}\right.$. We also note that $U_{0}^{0}(\theta, \phi)=1$ and $V_{0}^{0}(\theta, \phi)=0$. The square-root is employed to assure that the radius will be always nonnegative, and a small constant $\epsilon$ is added to ensure the differentiability of the radial function with respect to the shape parameters. It is important to note that this shape representation is not unique, since the same shape can be represented using a different center position and appropriately modified shape parameters. In matrix form, we have the following parametrization of the shape boundary:

$$
R(\theta, \phi, \mathbf{a})=\sqrt{\mathbf{B}^{T}(\theta, \phi) \mathbf{a}+\epsilon}
$$

where $\mathbf{B}$ is a matrix composed of our shape basis functions and $\mathbf{a}=\left[\begin{array}{lllllll}a_{0}^{0} & a_{1}^{0} & b_{1}^{0} & a_{1}^{1} & b_{1}^{1} & \ldots\end{array}\right]^{T}$.

In the results which follow, it will be necessary to compute the normal vector at each point of our parametrized shape's sur-

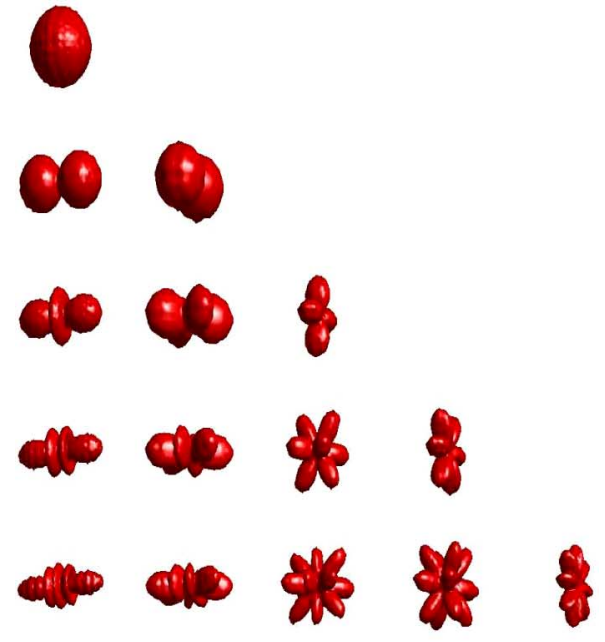

(a)

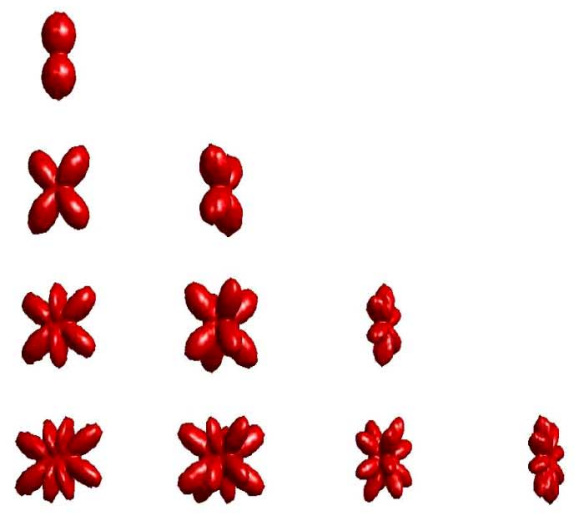

(b)

Fig. 1. Spherical Harmonics of up to fourth order. (a) Real part. (b) Imaginary part.

face. An analytical expression for this function can be derived by transformation to rectangular coordinates

$$
\begin{aligned}
& x=R(\theta, \phi, \mathbf{a}) \cos \phi \sin \theta \\
& y=R(\theta, \phi, \mathbf{a}) \sin \phi \sin \theta \\
& z=R(\theta, \phi, \mathbf{a}) \cos \theta .
\end{aligned}
$$

If we represent each point on the surface of the shape as $\mathbf{f}=$ $(x, y, z)$, then the normal vector, $\boldsymbol{\nu}$, can be computed as

where

$$
\boldsymbol{\nu}=\frac{\mathbf{f}_{\theta} \times \mathbf{f}_{\phi}}{\left\|\mathbf{f}_{\theta} \times \mathbf{f}_{\phi}\right\|_{2}}
$$

$$
\begin{aligned}
\mathbf{f}_{\theta}= & \left(\frac{\partial R(\theta, \phi, \mathbf{a})}{\partial \theta} \cos \phi \sin \theta+R(\theta, \phi, \mathbf{a}) \cos \phi \cos \theta\right. \\
& \frac{\partial R(\theta, \phi, \mathbf{a})}{\partial \theta} \sin \phi \sin \theta+R(\theta, \phi, \mathbf{a}) \sin \phi \cos \theta \\
& \left.\frac{\partial R(\theta, \phi, \mathbf{a})}{\partial \theta} \cos \theta-R(\theta, \phi, \mathbf{a}) \sin \theta\right) \\
\mathbf{f}_{\phi}= & \left(\frac{\partial R(\theta, \phi, \mathbf{a})}{\partial \phi} \cos \phi \sin \theta-R(\theta, \phi, \mathbf{a}) \sin \phi \sin \theta\right. \\
& \frac{\partial R(\theta, \phi, \mathbf{a})}{\partial \phi} \sin \phi \sin \theta+R(\theta, \phi, \mathbf{a}) \cos \phi \sin \theta \\
& \left.\frac{\partial R(\theta, \phi, \mathbf{a})}{\partial \phi} \cos \phi\right) .
\end{aligned}
$$


In order to compute $(\partial R(\theta, \phi, \mathbf{a}) / \partial \theta)$, it is useful to utilize the following identities:

$$
\begin{aligned}
& \frac{d P_{l}^{m}(\cos \theta)}{d \theta}= \\
& \begin{cases}\frac{l \cos \theta P_{l}^{m}(\cos \theta)-(l+m) P_{l-1}^{m}(\cos \theta)}{\sin \theta} & m<l \\
(-1)^{l}(2 l-1) !(l / 2)(2 \cos \theta \sin \theta)\left(\sin ^{2} \theta\right)^{\left(\frac{l}{2}-1\right)} & m=l\end{cases}
\end{aligned}
$$

\section{A. Centroid Constraint}

The shape parametrization described above is not unique, as a given shape can be described with respect to an arbitrary center point within its interior. To ensure uniqueness, we constrain our shape representation such that the center with respect to which it is defined is identical to the shape's centroid, which is unique and well-defined.

Thus, we impose the following constraints, which can be derived by integrating the expressions for the $x, y$, and $z$ coordinates of the estimated shape's centroid in spherical coordinates:

$$
\begin{aligned}
& \int_{0}^{2 \pi} \int_{0}^{\pi} R^{4}(\theta, \phi, \mathbf{a}) \cos \phi \sin ^{2} \theta d \theta d \phi=0 \\
& \int_{0}^{2 \pi} \int_{0}^{\pi} R^{4}(\theta, \phi, \mathbf{a}) \sin \phi \sin ^{2} \theta d \theta d \phi=0 \\
& \int_{0}^{2 \pi} \int_{0}^{\pi} R^{4}(\theta, \phi, \mathbf{a}) \cos \theta \sin \theta d \theta d \phi=0 .
\end{aligned}
$$

\section{Forward Modeling AND SENSITIVITy CALCUlations}

In a given DOT experiment, the medium is illuminated using modulated light by a point source using amplitude-modulated light and measurements are made at discrete detector positions, with detectors approximated as delta functions at the detector positions. The experiment is then repeated using a point source located at a different position. In what follows, we assume that all measurements are made in the frequency domain, using a single modulation frequency. We assume that photon propagation is well approximated by the diffusion equation [37] and make use of a zero partial-flux Robin-type boundary condition [38]. We also assume that the medium is piecewise constant and consists of two regions, $\Omega_{i}$ and $\Omega_{e}$, where $\Omega_{i}$ is simply connected, separated by an internal boundary $\Gamma_{i}$. We denote the exterior boundary by $\Gamma_{e}$. The photon intensity, $u_{s}(\mathbf{r})$ due to a source at $\mathbf{r}_{s}$ can be modeled as the solution of the following system of coupled partial differential equations:

$$
\begin{cases}-D_{e} \nabla^{2} u_{s}(\mathbf{r}, \omega)+\left[\mu_{a}^{e}-\frac{j \omega}{v}\right] u_{s}(\mathbf{r}, \omega)=0 & \mathbf{r} \varepsilon \Omega_{e} \\ \beta u_{s}(\mathbf{r}, \omega)+D_{e} \frac{\partial u_{s}(\mathbf{r}, \omega)}{\partial \boldsymbol{\nu}}=\delta\left(\mathbf{r}-\mathbf{r}_{s}\right) & \mathbf{r} \varepsilon \Gamma_{e} \\ -D_{i} \nabla^{2} u_{s}(\mathbf{r}, \omega)+\left[\mu_{a}^{i}-\frac{j \omega}{v}\right] u_{s}(\mathbf{r}, \omega)=0 & \mathbf{r} \varepsilon \Omega_{i}\end{cases}
$$

with the following boundary conditions on $\Gamma_{i}$ :

$$
\begin{cases}u_{s, e}(\mathbf{r}, \omega)=u_{s, i}(\mathbf{r}, \omega) & \mathbf{r} \varepsilon \Gamma_{i} \\ D_{e} \frac{\partial u_{s, e}(\mathbf{r}, \omega)}{\partial \boldsymbol{\nu}}=D_{i} \frac{\partial u_{s, i}(\mathbf{r}, \omega)}{\partial \boldsymbol{\nu}} & \mathbf{r} \varepsilon \Gamma_{i}\end{cases}
$$

where $D_{i}$ and $D_{e}$ are the diffusion coefficients in $\Omega_{i}$ and $\Omega_{e}$ respectively, $\mu_{a}^{i, e}$ are the absorption coefficients, the modulation frequency is $\omega, v$ is the speed of light in tissue, and the unit outward normal vector to a surface is $\nu$. The coefficient $\beta$ is dependent on the indices of refraction at the exterior boundary. The quantities $u_{s, i}(\mathbf{r})$ and $u_{s, e}(\mathbf{r})$ are the limits of $u_{s}(\mathbf{r})$ as we approach $\Gamma_{i}$ from the interior of the absorbing object and from its exterior, respectively.

We also consider the solution of the diffusion equation for a homogeneous medium, with a Robin-type source at the detector position $\mathbf{r}_{d}$

$$
\left\{\begin{array}{ll}
-D_{e} \nabla^{2} u_{d}(\mathbf{r}, \omega)+\left[\mu_{a}^{e}-\frac{j \omega}{v}\right] u_{d}(\mathbf{r}, \omega)=0 & \mathbf{r} \varepsilon \Omega_{e} \cup \Omega_{i} \\
\beta u_{d}(\mathbf{r}, \omega)+D_{e} \frac{\partial u_{d}(\mathbf{r}, \omega)}{\partial \boldsymbol{\nu}}=\delta\left(\mathbf{r}-\mathbf{r}_{d}\right) & \mathbf{r} \varepsilon \Gamma_{e}
\end{array} .\right.
$$

In the Appendix, it is shown that

$$
\begin{aligned}
& u_{s}\left(\mathbf{r}_{d}, \omega\right)-u_{d}\left(\mathbf{r}_{s}, \omega\right) \\
& =-\int_{\Omega_{i}} D \nabla u_{s}(\mathbf{r}, \omega) \cdot \nabla u_{d}(\mathbf{r}, \omega) d \mathbf{r} \\
& \quad-\int_{\Omega_{i}} \mu_{a} u_{s}(\mathbf{r}, \omega) u_{d}(\mathbf{r}, \omega) d \mathbf{r}
\end{aligned}
$$

where $D=D_{i}-D_{e}$ and $\mu_{a}=\mu_{a}^{i}-\mu_{a}^{e}$.

In our work, we assume that our measurements are comprised of delta functions at the detector locations. Thus, we define the source-detector measurement $h_{s, d}(\omega)=u_{s}\left(\mathbf{r}_{d}, \omega\right)$ as the measurement due to source $s$, measured by detector $d$. If we write (15) in polar coordinates, we obtain

$$
\begin{aligned}
h_{s, d}(\omega)-u_{d}\left(\mathbf{r}_{s}, \omega\right) \\
=-\mu_{a} \int_{0}^{2 \pi} \int_{0}^{\pi} \int_{0}^{R(\theta, \phi, \mathbf{a})} u_{s}(\theta, \phi, r, \omega) u_{d} \\
\quad \times(\theta, \phi, r, \omega) r^{2} \sin \theta d r d \theta d \phi \\
\quad-D \int_{0}^{2 \pi} \int_{0}^{\pi} \int_{0}^{R(\theta, \phi, \mathbf{a})} \nabla u_{s}(\theta, \phi, r, \omega) \\
\quad-\nabla u_{d}(\theta, \phi, r, \omega) r^{2} \sin \theta d r d \theta d \phi
\end{aligned}
$$

where the origin is taken to be the center of $\Omega_{i}$. In order to clearly illustrate the shape-based approach to image reconstruction, in the remainder of this paper we will consider reconstruction of objects which are absorbing only, for which $D=0$.

We now consider the calculation of the sensitivity functions required for both the optimization procedure discussed in Section IV and for the computation of performance bounds in Section VI. Derivatives of the observed data with respect to $\mathbf{a}$ and $\mu_{a}$ are obtained easily from (16) as

$$
\begin{aligned}
& \frac{\partial h_{s, d}(\omega)}{\partial a_{j}} \\
& =-\mu_{a} \int_{0}^{2 \pi} \int_{0}^{\pi} u_{s}[\theta, \phi, R(\theta, \phi, \mathbf{a}), \omega] u_{d} \\
& \quad \times[\theta, \phi, R(\theta, \phi, \mathbf{a}), \omega] \frac{\partial R(\theta, \phi, \mathbf{a})}{\partial a_{j}} \\
& \quad \times(R(\theta, \phi, \mathbf{a}))^{2} \sin \theta d \theta d \phi
\end{aligned}
$$




$$
\begin{aligned}
& \frac{\partial h_{s, d}(\omega)}{\partial \mu_{a}} \\
& =-\int_{0}^{2 \pi} \int_{0}^{\pi} \int_{0}^{R(\theta, \phi, \mathbf{a})} u_{s}(\theta, \phi, r, \omega) u_{d} \\
& \quad \times(\theta, \phi, r, \omega) r^{2} \sin \theta d r d \theta d \phi .
\end{aligned}
$$

We note that (17) is an integral over the surface of the object and (18) is an integral over the volume of the absorbing object.

To compute the derivative of the data with respect to the center position of the object, we make use shape derivative results in [29]. More specifically, if If $\mathbf{V}$ is a $C^{2}$ differentiable vector field defined in some neighborhood of $\Gamma_{i}$, then we can define the shape derivative of the field due to a particular point source with respect to an infinitesimal perturbation of $\Gamma_{i}$ in direction $\mathbf{V}$ as follows:

$$
\frac{\partial u_{s}\left(\Omega_{i}, \omega\right)}{\partial \mathbf{V}}=\lim _{\epsilon \rightarrow 0} \frac{u_{s}\left(\Omega_{i}+\epsilon \mathbf{V}, \omega\right)-u_{s}\left(\Omega_{i}, \omega\right)}{\epsilon} .
$$

Now note that for any function $f(\mathbf{r})$, if we define $y$ as $\int_{\Omega_{i}} f(\mathbf{r}) d \mathbf{r}$ then the shape derivative of $y$ with respect to a perturbation of $\Omega_{i}$ by $\mathbf{V}$ is [29]

$$
\frac{\partial y}{\partial \mathbf{V}}=\int_{\Gamma_{i}} f(s)(\mathbf{V}(\mathbf{s}) \cdot \boldsymbol{\nu}(\mathbf{s})) d \mathbf{s} .
$$

Making use of this expression, we can compute the sensitivity of the measurements to perturbations in the object's center position. Perturbing the $x$ coordinate of our object's center is equiva-

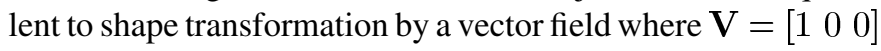
everywhere. Thus, the derivative of a measurement with respect to a change in the $x$ component of the center position can be computed as

$$
\frac{\partial h_{s, d}(\omega)}{\partial c_{x}}=-\mu_{a} \int_{\Gamma_{i}} u_{s}(\mathbf{s}, \omega) u_{d}(\mathbf{s}, \omega) \nu_{x} d \mathbf{s} .
$$

The derivatives with respect to changes in the $y$ and $z$ components of the object center position are found analogously. The gradient with respect to the object center position, $\mathbf{c}$, is then

$$
\nabla_{\mathbf{c}} h_{s, d}(\omega)=-\mu_{a} \int_{\Gamma_{i}} u_{s}(\mathbf{s}, \omega) u_{d}(\mathbf{s}, \omega) \boldsymbol{\nu} d \mathbf{s} .
$$

\section{Cost Function AND OptimizATION}

In a typical DOT experiment, the medium is illuminated sequentially at each of $M$ source positions, with measurements made at $N$ detector positions. If we arrange our measurements as a vector, the goal of our inverse problem is to minimize the following cost function, where we assume that all measurements are made at a given discrete modulation frequency:

$$
\begin{aligned}
\hat{\mathbf{p}} & =\arg \min _{\mathbf{p}}\|\mathbf{y}-\mathbf{h}(\mathbf{p})\|_{\Sigma_{n}^{-1}}^{2} \\
\mathbf{y} & =\left[\left|y_{1,1}\right| \cdots\left|y_{M, N}\right| \operatorname{Arg}\left(y_{1,1}\right) \cdots \operatorname{Arg}\left(y_{M, N}\right)\right]^{T} \\
\mathbf{h} & =\left[\left|h_{1,1}\right| \cdots\left|h_{M, N}\right| \operatorname{Arg}\left(h_{1,1}\right) \cdots \operatorname{Arg}\left(h_{M, N}\right)\right]^{T}
\end{aligned}
$$

where $\left|y_{i, j}\right|$ and $\operatorname{Arg}\left(y_{i, j}\right)$ are the magnitude and phase, respectively, of the measurement of the fluence due to source $i$, measured at detector location $j .\left|h_{i, j}\right|$ and $\operatorname{Arg}\left(h_{i, j}\right)$ are the corresponding hypothesized measurements of magnitude and phase, given a forward solver and an estimate of the object shape parameters, $\mathbf{p}$. The covariance matrix of the noise, assumed to be Gaussian, is given by $\Sigma_{n}$.

In this paper, $\mathbf{p}$ has the following form:

$$
\mathbf{p}=\left[\begin{array}{lllll}
c_{x} & c_{y} & c_{z} & \mu_{a} & \mathbf{a}^{T}
\end{array}\right]^{T}
$$

where $c_{x}, c_{y}, c_{z}$ are the $x, y$, and $z$ components of the object center position, respectively, $\mu_{a}$ is the absorption contrast, and $\mathbf{a}$ is the shape parameter vector.

In the shape optimization, we make use of a projected Newton method [39], which is the projection of the Levenberg-Marquardt method onto the manifold defined by (9)-(11). Specifically, we employ the following algorithm.

\section{Algorithm 1:}

1) Choose values for $\mathbf{c}_{0}$, the initial center position, $\mu_{a, 0}$, the initial contrast, and $\mathbf{a}_{0}$, the initial shape parameter vector.

2) Compute the source and detector solutions for the current shape estimate: $\mathbf{c}_{i}, \mu_{a, i}, \mathbf{a}_{i}$.

3) Compute the cost function

$$
C=\left\|\mathbf{y}-\mathbf{h}\left(\mathbf{c}_{i}, \mu_{a, i}, \mathbf{a}_{i}\right)\right\|_{\mathbf{\Sigma}_{n}^{-1}} .
$$

4) Compute $\mathbf{J}_{i}=\left[\begin{array}{lll}\mathbf{J}_{\mathbf{c}, i} & \mathbf{J}_{\mu_{a, i}} & \mathbf{J}_{\mathbf{a}, i}\end{array}\right]$.

5) Update the estimate

$$
\begin{aligned}
& {\left[\begin{array}{lll}
\mathbf{c}_{i+1} & \mu_{a, i+1} & \mathbf{a}_{i+1}^{T}
\end{array}\right]^{T}=\left[\begin{array}{lll}
\mathbf{c}_{i} & \mu_{a, i} & \mathbf{a}_{i}^{T}
\end{array}\right]^{T}} \\
& \quad+\int_{0}^{S} \mathbf{U}(s)\left(\mathbf{U}^{T}(s) \mathbf{J}_{i}^{T} \boldsymbol{\Sigma}_{n}^{-1} \mathbf{J}_{i} \mathbf{U}(s)+\lambda I\right)^{-1} \\
& \quad \times \mathbf{U}^{T}(s) \mathbf{J}_{i}^{T} \boldsymbol{\Sigma}_{n}^{-1}\left(\mathbf{y}-\mathbf{h}\left(\mathbf{c}_{i}, \mu_{a, i}, \mathbf{a}_{i}^{T}\right)\right) d s
\end{aligned}
$$

where we choose $\lambda$ and $S$ to minimize the cost function of the new estimate. We choose $S$ by means of a backtracking line search [40].

6) Return to step 2 until convergence.

In (27), $\mathbf{U}(t)$ is an orthonormal basis for the null space of the gradient matrix of the constraint set defined by (9)-(11), and $S$ is the length of the step taken in the search direction. The Jacobian matrices, $\mathbf{J}_{\mathbf{c}, i}, \mathbf{J}_{\mu_{a, i}}$, and $\mathbf{J}_{\mathbf{a}, i}$ are obtained by computing (22), (18), and (17), respectively, for all source-detector measurement pairs. We note that we assume that $\mathbf{y}$ and $\mathbf{h}$ are comprised of measurements of amplitude and phase and we make use of the chain rule in order to compute the Jacobian matrices with respect to these measurement types. We approximate the integral in (27) numerically. This optimization is an extension of the standard Levenberg-Marquardt method, where the search direction is projected onto a lower-dimensional manifold defined by the constraint set.

For the purpose of comparison, we also make use of an algorithm in which the object center position is known a priori. In this case, we use an unconstrained Levenberg-Marquardt algorithm and optimize only the shape parameters and the object contrast.

Algorithm 2:

1) Choose values for $\mu_{a, 0}$, the initial contrast, and $\mathbf{a}_{0}$, the initial shape parameter vector.

2) Compute the source and detector solutions for the current shape estimate: $\mu_{a, i}, \mathbf{a}_{i}$

3) Compute the cost function: $C=\left\|\mathbf{y}-\mathbf{h}\left(\mu_{a, i}, \mathbf{a}_{i}\right)\right\|_{\boldsymbol{\Sigma}_{n}^{-1}}$ 
4) Compute $\mathbf{J}_{i}=\left[\mathbf{J}_{\mu_{a, i}} \mathbf{J}_{\mathbf{a}, i}\right]$

5) Update the estimate:

$$
\begin{aligned}
& {\left[\begin{array}{ll}
\mu_{a, i+1} & \mathbf{a}_{i+1}^{T}
\end{array}\right]^{T}=\left[\begin{array}{ll}
\mu_{a, i} & \mathbf{a}_{i}^{T}
\end{array}\right]^{T}} \\
& \quad+S\left(\mathbf{J}_{i}^{T} \boldsymbol{\Sigma}_{n}^{-1} \mathbf{J}_{i}+\lambda I\right)^{-1} \mathbf{J}_{i}^{T} \boldsymbol{\Sigma}_{n}^{-1}\left(\mathbf{y}-\mathbf{h}\left(\mu_{a, i}, \mathbf{a}_{i}\right)\right)
\end{aligned}
$$

where we choose $\lambda$ and $S$ to minimize the cost function of the new estimate. We choose $S$ by means of a backtracking line search.

6) Return to step 2 until convergence.

\section{VOXEL-BASED NONLINEAR RECONSTRUCTION}

We have also implemented a more standard image reconstruction algorithm in which we estimate the absorption at each point in space by assuming that it can be decomposed as a linear combination of piecewise-constant basis functions

$$
\mu_{a}(\mathbf{r})=\sum_{n=1}^{N} \chi_{n}(\mathbf{r}) v_{n}
$$

where $\chi_{n}(\mathbf{r})$ is the characteristic function of voxel $n$.

Estimating the absorption at each point in space, given this assumption, is then equivalent to estimating the vector $\mathbf{v}=$ $\left[\begin{array}{llll}v_{1} & v_{2} & \ldots & v_{n}\end{array}\right]^{T}$. It can be shown that the Fréchet derivative of a measurement $h_{s, d}$ with respect to a small perturbation in $\mathbf{v}$ is [10]

$$
\frac{\partial h_{s, d}}{\partial \mathbf{v}}=\sum_{n=0}^{N} \delta v_{n} \int_{\chi_{n}} u_{s}(\mathbf{r}) u_{d} d \mathbf{r} .
$$

We then make use of a nonlinear optimization algorithm to estimate $\mathbf{v}$, with Tikhonov regularization applied to stabilize the inversion. Our optimization algortithm, then, strives to minimize the following quadratic cost function:

$$
C(\mathbf{v})=\|\mathbf{y}-\mathbf{h}(\mathbf{v})\|_{\Sigma_{n}^{-1}}^{2}+\lambda\|\mathbf{L v}\|_{2}^{2}
$$

where $\lambda$ is the regularization parameter and $\mathbf{L}$ is the finite-difference approximation of the Laplacian [9], used to introduce smoothing of $\mathbf{v}$. We implement $\mathbf{L}$ as a sparse matrix. Specifically, we follow the unconstrained Gauss-Newton procedure listed below.

\section{Algorithm 3:}

1) Choose a value for $\mathbf{v}_{0}$, the initial guess. As our initial guess, we assume that $\mathbf{v}=v\left[\begin{array}{llll}1 & 1 & \ldots & 1\end{array}\right]^{T}$, where $v$ is known $a$ priori.

2) Compute the source and detector solutions for the current value of $\mathbf{v}$.

3) Compute the cost function

$$
C=\|\mathbf{y}-\mathbf{h}(\mathbf{v})\|_{\Sigma_{n}^{-1}}^{2}+\lambda\|\mathbf{L v}\|_{2}^{2} .
$$

4) Compute the Jacobian matrix $\mathbf{J}_{\mathbf{v}, i}$, using (30) and applying the chain rule to compute the sensitivity with respect to amplitude and phase measurements, for all source-detector measurement pairs.
5) Update the estimate

$$
\begin{aligned}
\mathbf{v}_{i+1}=\mathbf{v}_{i}+S\left(\mathbf{J}_{i}^{T}\right. & \left.\boldsymbol{\Sigma}_{n}^{-1} \mathbf{J}_{i}+\lambda \mathbf{L}^{T} \mathbf{L}\right)^{-1} \\
& \times\left[\mathbf{J}_{i}^{T} \boldsymbol{\Sigma}_{n}^{-1}\left(\mathbf{y}-\mathbf{h}\left(\mathbf{v}_{\mathbf{i}}\right)\right)-\lambda \mathbf{L}^{T} \mathbf{L} \mathbf{v}_{\mathbf{i}}\right]
\end{aligned}
$$

where we choose $S$ to minimize the cost function of the new estimate. We choose $S$ by means of a backtracking line search.

6) Return to step 2 until convergence.

\section{CRAMÉR-RAO LOWER BOUND AND SHAPE BOUNDS}

An advantage of being able to explicitly compute shape sensitivities is that we can compute a lower bound on shape parameter estimation accuracy, in the case of an unbiased estimator, which we will be assume to be the case for our estimation procedure. It is possible to extend the approach used in this section to biased estimators [41] and to Bayesian estimation [42].

More specifically, we can compute a lower bound on the estimation covariance using the Fisher information matrix (FIM):

$$
E_{\mathbf{p}}\left[(\hat{\mathbf{p}}-\mathbf{p})(\hat{\mathbf{p}}-\mathbf{p})^{T}\right] \geq \mathbf{F}_{\mathbf{p}}^{-1}
$$

where the FIM is

$$
\mathbf{F}_{\mathbf{p}}=\left[\left(\nabla_{\mathbf{p}} \ln f(\mathbf{y}, \mathbf{p})\right)\left(\nabla_{\mathbf{p}} \ln f(\mathbf{y}, \mathbf{p})\right)^{T}\right]
$$

and $f(\mathbf{y}, \mathbf{p})$ is the joint probability density of the observation vector $\mathbf{y}$ and the parameter vector $\mathbf{p}$ where $E[\hat{\mathbf{p}}]$ denotes the expected value of our estimate.

In the case of measurements corrupted by Gaussian noise, we have the following simplified form for the FIM for the estimation of a:

$$
\mathbf{F}_{\mathbf{p}}=\mathbf{J}_{\mathbf{p}}^{T} \boldsymbol{\Sigma}_{n}^{-1} \mathbf{J}_{\mathbf{p}}
$$

where $\mathbf{J}_{\mathbf{p}}$ is the Jacobian of our measurements with respect to $\mathbf{p}$, each element of which can be computed using (17), (18), and (22).

In our case, where there are additional constraints on $\mathbf{p}$, it is necessary to use the constrained Cramér-Rao bound. If $\mathbf{c}(\mathbf{p})$ is a set of differentiable constraints and $\mathbf{J}_{\mathbf{c}}(\mathbf{p})$ is its gradient matrix, then it can be shown that [35]

$$
E_{\mathbf{p}}\left[(\hat{\mathbf{p}}-\mathbf{p})(\hat{\mathbf{p}}-\mathbf{p})^{T}\right] \geq \mathbf{F}_{\mathbf{p}}^{c}
$$

where the constrained Fisher information matrix is

$$
\mathbf{F}_{\mathbf{p}}^{c}=\mathbf{U}\left(\mathbf{U}^{T} \mathbf{F}_{\mathbf{p}} \mathbf{U}\right)^{-1} \mathbf{U}^{T}
$$

and $\mathbf{U}$ is a matrix whose columns form an orthonormal basis for the null-space of $\mathbf{J}_{\mathbf{c}}(\mathbf{p})$.

Given a lower bound for the parameter covariance matrix, $E_{\mathbf{p}}\left[(\hat{\mathbf{p}}-\mathbf{p})(\hat{\mathbf{p}}-\mathbf{p})^{T}\right]$ we wish to determine bounds on the shape itself. To accomplish this end, for each point on the boundary, we compute the maximum deviation such that the parameter vector is within a given confidence interval. Thus, we solve the following constrained optimization problem for each point on the boundary:

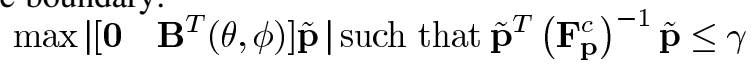




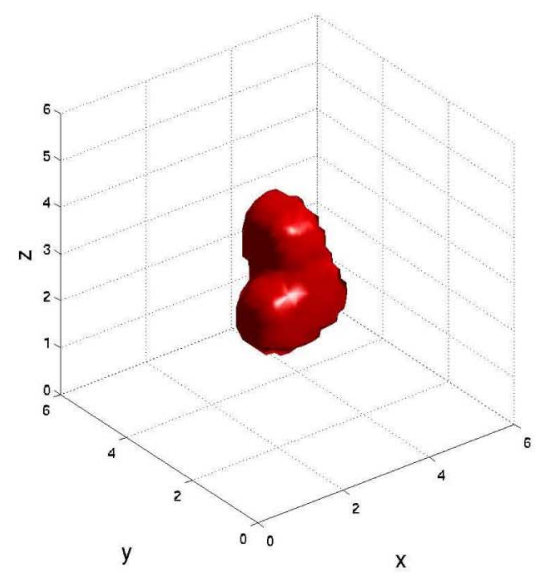

(a)
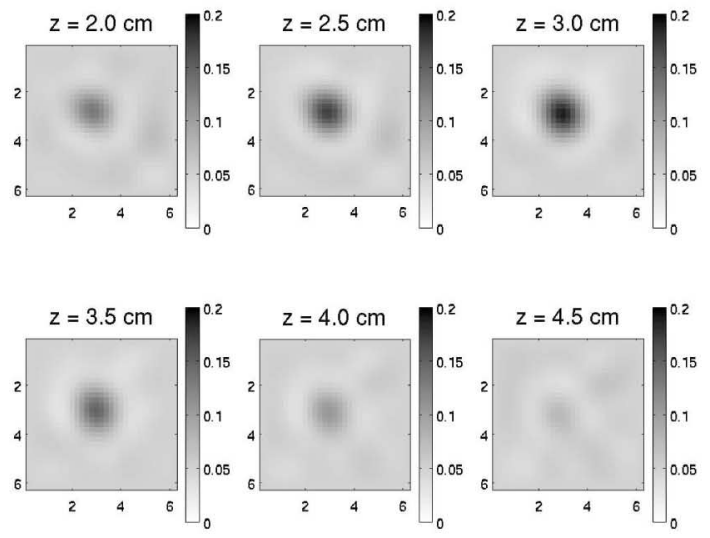

(c)

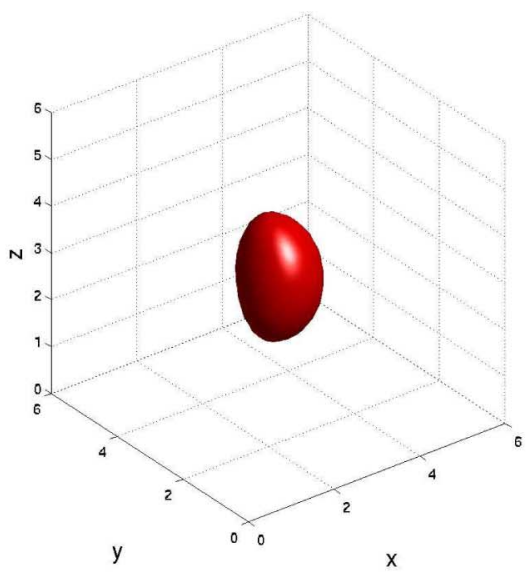

(b)

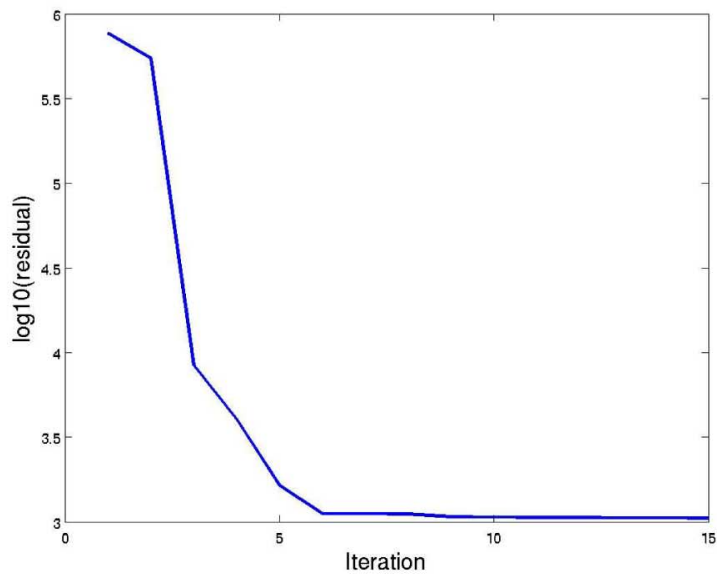

(d)

Fig. 2. Randomly generated third-order shape and performance of a voxel-wise algorithm in reconstructing this shape. (a) True third-order absorbing object. (b) Fifty percent absorption isosurface for the voxel-wise reconstruction. (c) Slices in $z$ for voxel-wise reconstruction. (d) Convergence of the cost function for the voxel-wise reconstruction.

where $\gamma$ is our confidence interval. In this equation, the $\mathbf{0}$ block, with dimension $1 \times 4$ is used to select only those shape parameters used to encode the object radius, ignoring those which relate to object position and contrast. This expression can be used to computed the maximal perturbation in the object radius, where $\tilde{\mathbf{p}}=\hat{\mathbf{p}}-\mathbf{p}$. The same procedure can be used to give us a confidence interval on the object center position and on the contrast.

\section{RESULTS AND DISCUSSION}

In order to evaluate the performance of our algorithms, we tested them for randomly-generated objects with predefined levels of complexity. These objects were assumed to be parameterized using (3), where $a_{0}^{0}$ was $2 \mathrm{~cm}$ and the remaining coefficients of a were drawn from a multivariate Gaussian prior distribution with covariance $\sigma_{\mathbf{a}}^{2} \mathbf{I}$ where $\sigma_{\mathbf{a}}^{2}$ was 0.1 . In all of the simulations that follow, we assumed that the measurement noise was Gaussian, with a diagonal covariance matrix. The components of the noise covariance matrix for amplitude measurements were proportional to the measured amplitudes (with the constant of proportionality chosen to achieve a desired signal to noise ratio) and the diagonal elements of the covariance matrix corresponding to phase measurements all had the same value.
In Fig. 2(a), we show a shape generated using spherical harmonic coefficients of order 3 and below, for which we have simulated diffuse optical measurements. The object is embedded in a cubical region filled with a diffusing medium $6 \mathrm{~cm}$ on a side, and we make use of a transmission geometry, with 25 sources and 25 detectors uniformly distributed on the $z=0 \mathrm{~cm}$ and $z=6 \mathrm{~cm}$ planes, respectively. A background absorption of 0.05 $\mathrm{cm}^{-1}$ was assumed, and we assumed an absorption contrast of $0.15 \mathrm{~cm}^{-1}$ The true absorber was centered at $(3,3,3) \mathrm{cm}$ and had a volume of $2.94 \mathrm{~cm}^{3}$. We initialized our reconstructions with a spherical object $1.6 \mathrm{~cm}$ in diameter centered at $(4,4,4)$ $\mathrm{cm}$, with an absorption contrast of $0.07 \mathrm{~cm}^{-1}$.

We have empirically found that the following procedure is quite effective in simultaneously estimating an object's shape and contrast. We first assume that the object is spherical (i.e., using a spherical harmonic representation of order 0). After the zeroth-order estimate has converged, we initialize a higherorder model with the position estimated using the simplified spherical model. Skipping this initialization step tends to significantly reduce the convergence rate of our algorithms or will even cause them to converge to suboptimal local minima.

In all of the examples described in this paper, we make use of a finite-difference-based forward model, discretized using a uni- 


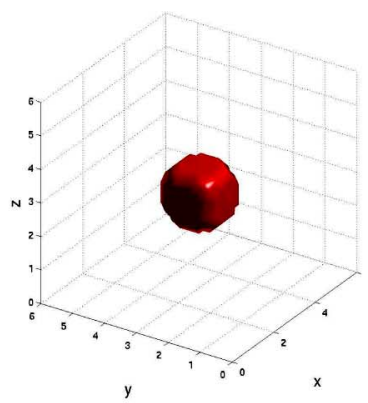

(a)

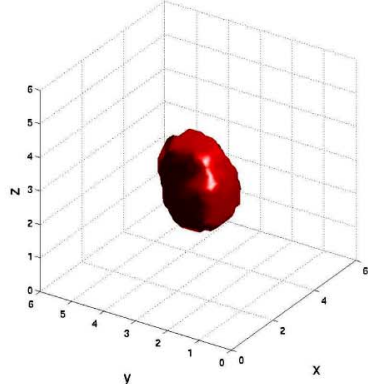

(b)

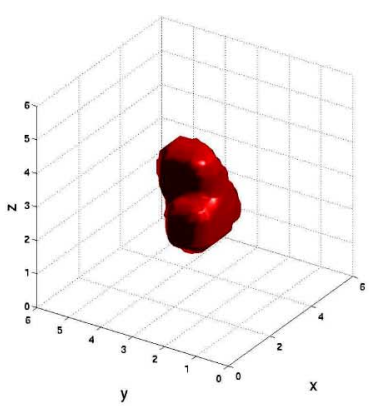

(c)

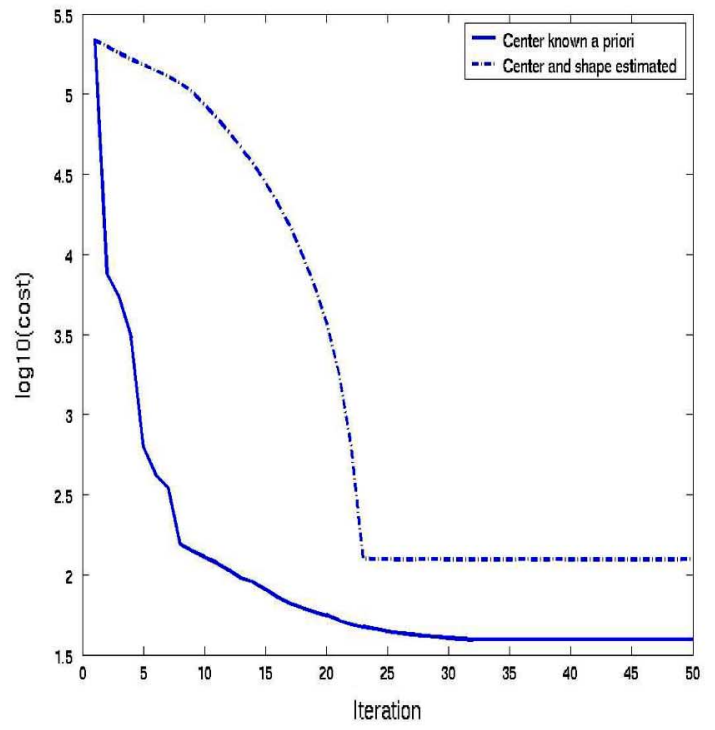

(d)

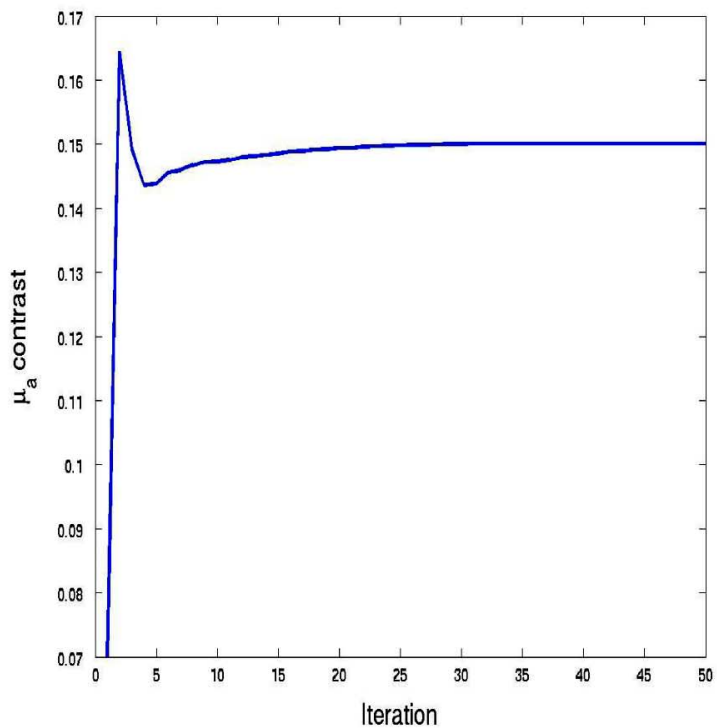

(e)

Fig. 3. Evolution of third-order shape estimate and performance of shape-based reconstruction algorithm. (a) Iteration 1. (b) Iteration 3. (c) Iteration 5. (d) Convergence of the cost function. (e) Convergence of the absorption contrast estimate.

form grid, with voxels $0.2 \mathrm{~cm}$ on a side. Partial-volume effects are taken into account by computing the volume of the intersection of the object with each voxel, making use of integration in spherical coordinates. To compute the forward solution, we discretize the partial differential equation (PDE) described by (12) as a large, sparse matrix, and solve using the GMRES iterative method [43]. A modulation frequency of $70 \mathrm{MHz}$ was used for all measurements.

For the purpose of comparison, we show in Fig. 2(b) and (c) a voxel-based nonlinear reconstruction of the absorption, computed using Algorithm 3, for data produced by the absorbing object shown in Fig. 2(a). For both the voxel-based and shape-based reconstructions, amplitude-dependent Gaussian noise was added such that the signal-to-noise ratio (SNR) of the amplitude measurements was $80 \mathrm{~dB}$, and Gaussian noise with a standard deviation of $0.1^{\circ}$ was added to the phase measurements. For all three optimization algorithms, we chose as a stopping criterion that the change in the residual between two iterations of a given algorithm be less than $1 \%$. The regularization parameter was estimated by means of the L-curve method [44], [45] to have a value of 1.0. We note that the peak absorption contrast is estimated to within $10 \%$ of its true value, but, as is generally the case with DOT reconstructions, the reconstructed absorber is quite smooth and is lacking distinct edges. Thus, if the reconstructed image in Fig. 2(b) represented a tumor, it would be very difficult to definitively estimate the tumor's volume, or for that matter, its actual absorption contrast.

In Fig. 2(d), we show the convergence behavior of our voxelwise reconstruction procedure, as a function of iteration, noting that the cost function which we will reference in the remainder of this paper is the square-root of (23). Fig. 2(b) shows the $50 \%$ isosurface of the reconstructed absorber. Reducing the regularization parameter may help to reconstruct missing fine details, but the level of image artifacts worsens.

In contrast, the results of the shape-based image reconstruction, using the same data as for the voxel-wise reconstruction, are shown in Fig. 3, which illustrates the evolution of our shape estimation in the first five iterations of the algorithm. We note that, for this case, at least visually, the true shape, position, and orientation of the absorber are reconstructed quite accurately after five iterations of the algorithm. The initialization step of the algorithm, using a zeroth-order model, converged from an initial guess of $(4,4,4) \mathrm{cm}$ to a center position estimate of $(3.18$, $3.20,3.28) \mathrm{cm}$ in 15 iterations. After full optimization, in which all parameters were allowed to vary, the final center position estimate was $(3.09,2.89,3.03) \mathrm{cm}$. As illustrated in Fig. 3(d), the cost function continued to decrease further until approximately 


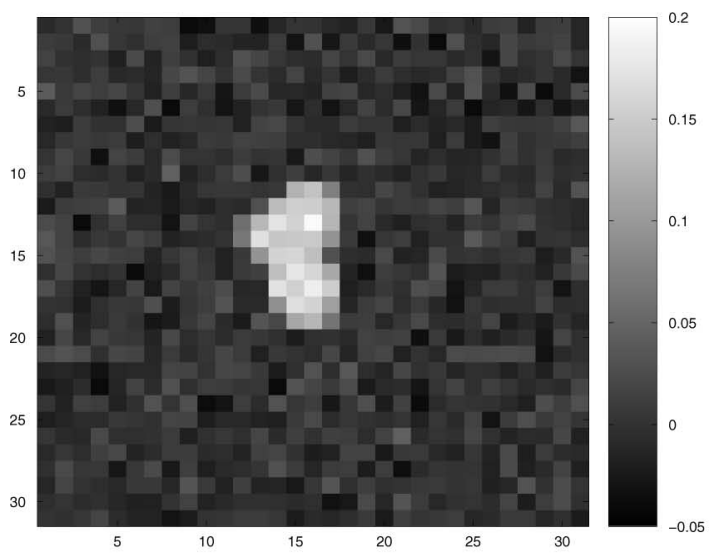

(a)

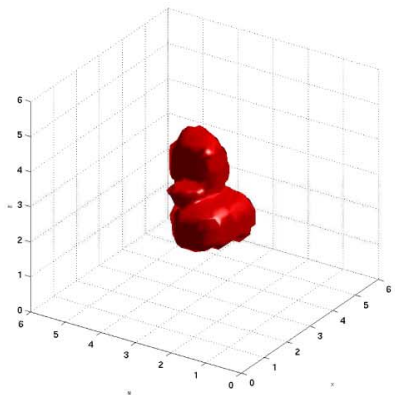

(c)

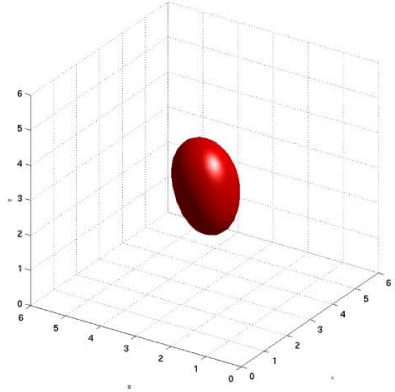

(e)

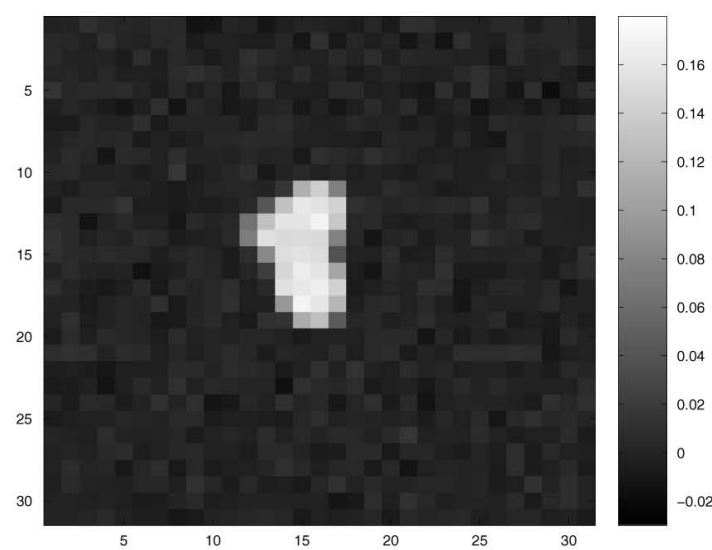

(b)

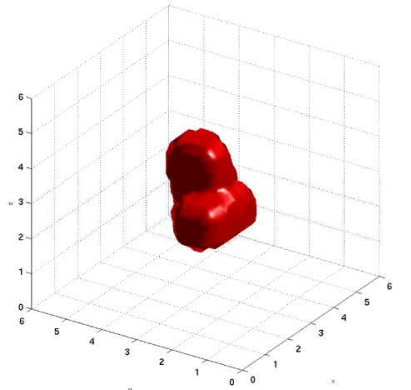

(d)

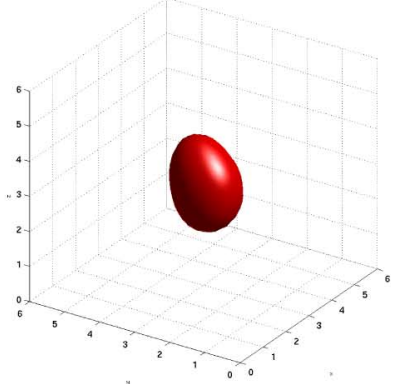

(f)

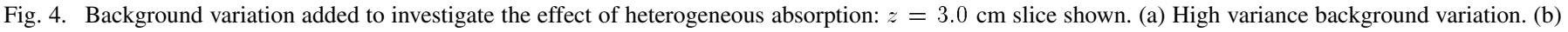

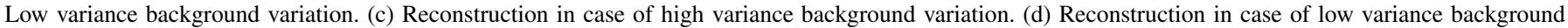

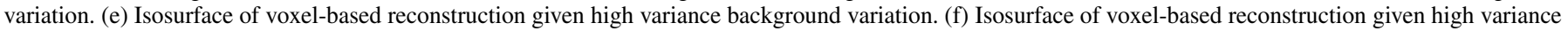
background variation.

the twenty-fifth iteration. Fig. 3(e) shows that an absorption contrast of $0.14 \mathrm{~cm}^{-1}$ is estimated after five iterations, but that the convergence rate for the contrast drops at this point, requiring approximately 20 more iterations for the contrast to converge to its true value of $0.15 \mathrm{~cm}^{-1}$. This is typical in our simulations; there seems to be a subtle interplay between accurate estimation of shape and estimation of contrast.

Fig. 3(d) also compares the convergence of the algorithm in the cases where the object center position is known a priori to the case when it is not assumed to be known. In the former case, Algorithm 2 is used, and, in the latter Algorithm 1 is employed. In both cases, the algorithm converges to a cost considerably lower than the minimum achieved by the voxel-based reconstruction approach. In addition, as expected, the rate of convergence is somewhat slower in the case where we have less $a$ priori information, but the minimum is still reached in approximately 25 iterations. In the case where the center position was known, our estimator was able to reach a value within one standard deviation of the expected Chi-squared error due to noise, meaning that essentially all of the information was extracted from the measurements. This minimum was not reached in the case where the center was not assumed known, and the reason for this is that our projected-Newton algorithm did not satisfy the centroid constraint exactly, and thus the estimated center tended to drift slightly from the true center position as the algorithm progressed (with the shape parameters compensating accordingly).

In terms of computation time, each iteration of algorithms 1,2 , and 3 requires approximately the same amount of time to complete, as computation of the shape and voxel-based Jacobian matrices is very nearly instantaneous compared to the time required for solution of the forward problem. Algorithms 1 and 2, which required approximately 25 and 30 iterations to converge, respectively, used approximately $24 \mathrm{~h}$ of computation 


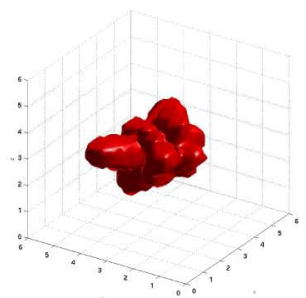

(a)

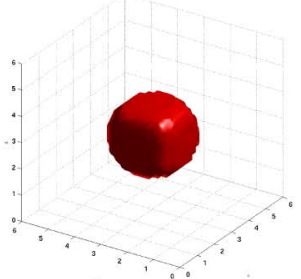

(b)

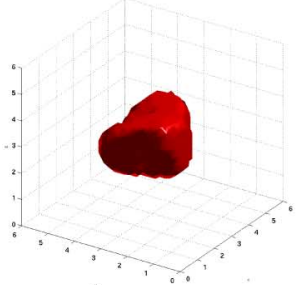

(e)

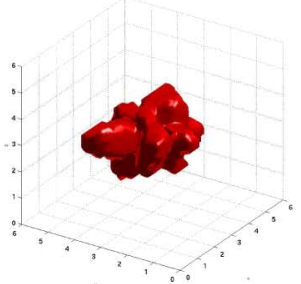

(h)

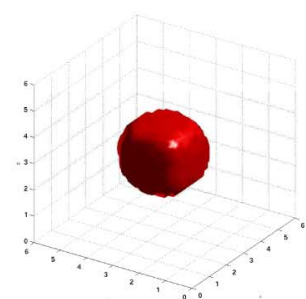

(c)

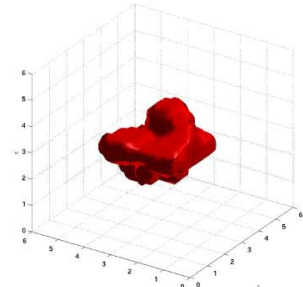

(f)

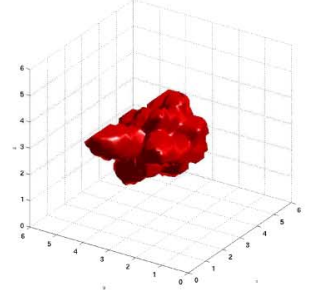

(i)

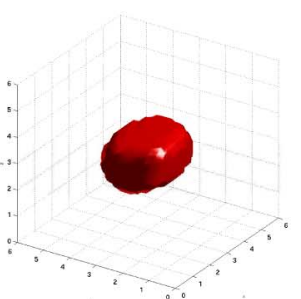

(d)

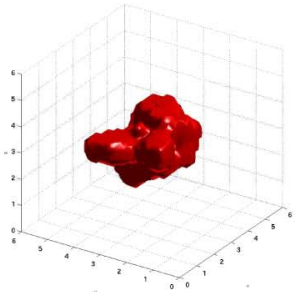

(g)

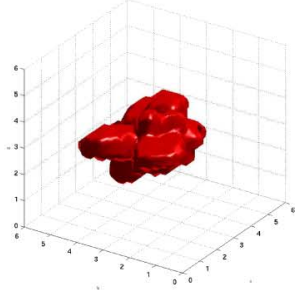

(j)

Fig. 5. Shape reconstruction as a function of model order: (a) true eighth-order absorbing object; (b) order 0 estimate; (c) order 1; (d) order 2; (e) order 3; (f) order $4 ;(\mathrm{g})$ order 5 ; (h) order 6; (i) order 7; (j) Order 8.

time on an AMD Opteron workstation with 2 GB of memory. Each forward/adjoint solve required approximately five minutes of computation time and much of the computational cost of our algorithms resulted from the use of a fairly precise line search in the update step of the Newton method. In a limited set of simulation studies, we have found that an equally accurate solution can be found with a much less accurate, and, thus, much less computationally expensive, line search. In contrast, algorithm 3 converged to a less accurate solution in six iterations, consuming approximately $6 \mathrm{~h}$ of computation time on a workstation with the same characteristics.

An important question is whether shape-based image reconstruction methods are robust even in the very realistic circumstance where the medium is not exactly piecewise constant. To explore this question, we have conducted simulations with variation in the medium's background absorption coefficient, where, again, the true absorbing object is shown in Fig. 2(a). We generated Gaussian independent identically distributed random noise fields with two different values chosen for the noise variance. These random noise fields were added to the absorption throughout the image, including within the absorbing anomaly, with the resulting absorption images for the $z=3.0 \mathrm{~cm}$ slice of the medium shown in Fig. 4(a) and (b). In Fig. 4(a) and (b), we have simulated random processes with relatively high and low variance, with standard deviations of $0.03 \mathrm{~cm}^{-1}$ and $0.005 \mathrm{~cm}^{-1}$, respectively, where the background absorption was $0.05 \mathrm{~cm}^{-1}$. The results of shape-based reconstructions, using Algorithm 1, for the levels of background variation in Fig. 4(a) and (b) are shown in Fig. 4(c) and (d), respectively. The volume of the actual object, shown in Fig. 2(a), is $2.94 \mathrm{~cm}^{3}$. For the case of low-variance background variation, the estimated volume was $2.6 \mathrm{~cm}^{3}$ and the estimated absorption contrast was $0.17 \mathrm{~cm}^{-1}$ (the true contrast was 0.15 $\mathrm{cm}^{-1}$ ), giving errors of $-11.6 \%$ and $13.3 \%$ in volume and contrast, respectively. In the case of high-variance background absorption variation, the errors in estimated object volume and contrast were $-26.5 \%$ and $33.3 \%$, respectively.

For the purpose of comparison, we have computed voxelbased reconstructions of absorption, using Algorithm 3, for the cases shown in Fig. 4(a) and (b). The isosurfaces representing a 
value of absorption $50 \%$ below the peak perturbation are shown in Fig. 4(e) and (f), respectively. We note that the voxel-based reconstruction algorithm estimated approximately the correct location, contrast (in both cases, a peak contrast of $0.15 \mathrm{~cm}^{-1}$ was estimated), and orientation for the absorbing object, but, again, the reconstructions were excessively smooth and the true shape of the boundary was not recovered.

Next we study model-order selection while reporting the performance of Algorithm 1 for a more complex shape. We also increased the amplitude and phase noise such that the amplitude SNR was only $40 \mathrm{~dB}$ and the standard deviation of the phase noise was $1.0^{\circ}$. Fig. 5(a) shows a randomly-generated shape, using spherical harmonic coefficients of order 8 and below. This example is intended to model the spiculated nature of tumors in the breast. Fig. 5(b)-(j) shows the estimated shape as we vary the order of the model used in the reconstruction. As in the previous example, the absorption contrast was $0.15 \mathrm{~cm}^{-1}$. We note that we are able to reconstruct the fine details of the absorber shape, although it is not visually clear whether there is reconstruction accuracy improvement for models of order 5 and higher.

In Fig. 6, we report a quantitative analysis of the results shown in Fig. 5. In Fig. 6(a), we see that the cost function continues to decrease as the model-order used in the reconstruction is increased until order 6 . For orders 6 and above, the cost is approximately constant. Fig. 6(b) shows that the image reconstruction error also decreases as the reconstruction model-order increases until order 6, but that the error increases using models of orders 7 and 8 . Thus, in higher noise situations, using an excessively complex model can be counter-productive. Fig. 6(c) and (d) shows the estimation of object volume and contrast as a function of model order, with the true volume and contrast, respectively, depicted as dashed lines. By numerical integration, we computed a true inhomogeneity volume of $4.82 \mathrm{~cm}^{3}$ and, using a model of order 6 , which minimized the reconstruction error, the estimated volume was $4.43 \mathrm{~cm}^{3}$, giving an error of $-8.1 \%$. Likewise, for the order 6 model, the estimated contrast was $0.164 \mathrm{~cm}^{-1}$, an overestimation of the true contrast by $9.3 \%$. Even in the case of a relatively high level of measurement noise, then, the volume and contrast of a high-contrast absorber with a very complex shape can be estimated quite accurately, with errors of less than $10 \%$ in object volume and absorption contrast. Fig. 6(e) shows the interesting result that, regardless of the model complexity used in the reconstruction, the product of the estimated contrast and the estimated object volume is approximately constant, as suggested by the analysis in [46].

Finally, we visualize Cramér-Rao-based shape recontruction bounds for the object shown in Fig. 2. In Fig. 7, we visualize the shape reconstruction uncertainty region assuming amplitude SNR of $80 \mathrm{~dB}$ and phase noise having a standary deviation of $1^{\circ}$. The bounds are computed using (38), with $\gamma=0.95$. In Fig. 7(a), we show the reconstructed shape bounds where the center position and object contrast are known a priori, corresponding to Algorithm 2. In Fig. 7(b), the same results are shown where the center position and object contrast are not known a priori, corresponding to Algorithm 1, and the centroid constraints, (9)-(11) are imposed. In this case, the constrained Cramér-Rao bound must be used. We observe that the unknown center position and unknown contrast do increase the maximal (a)

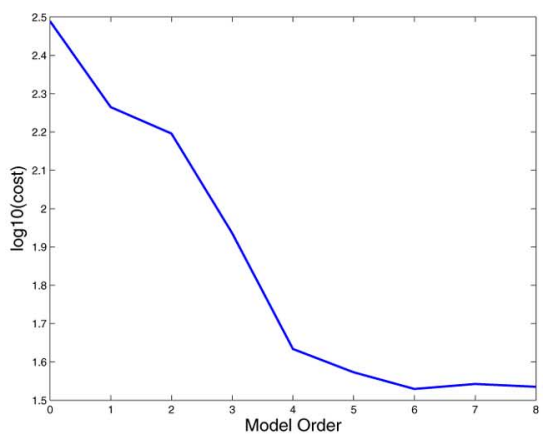

(b)

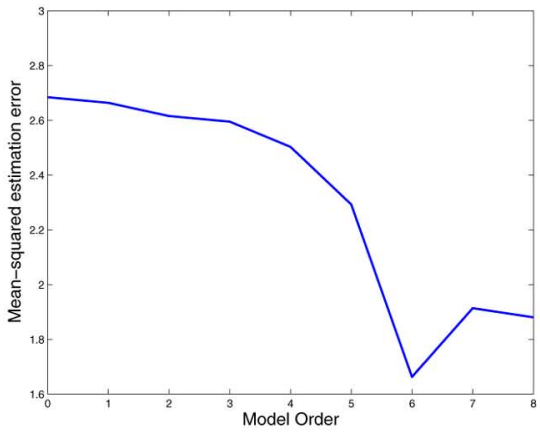

(c)

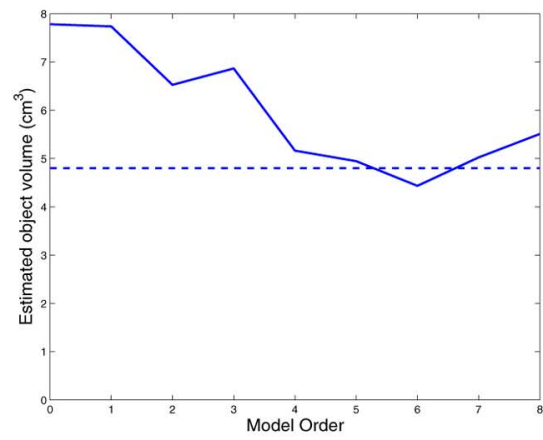

(d)

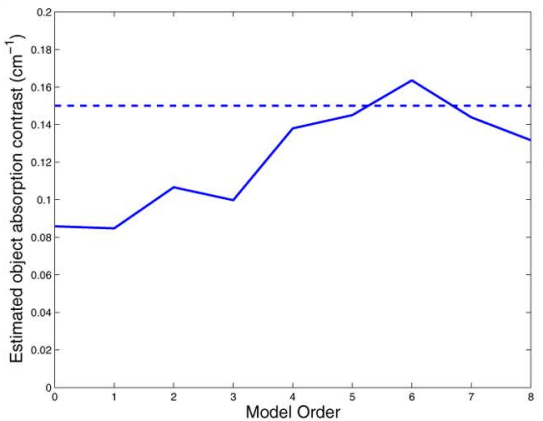

(e)

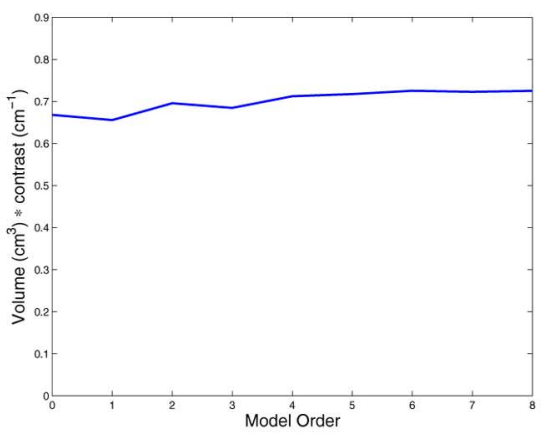

Fig. 6. Quantitative investigation of model order. (a) Residual at estimate to which shape reconstruction converged, as a function of model order. (b) Reconstruction error as a function of model order. (c) Estimated shape volume as a function of model order. (d) Estimated absorption contrast as a function of model order. (e) Product of volume and absorption contrast for estimated shape as a function of model order. 


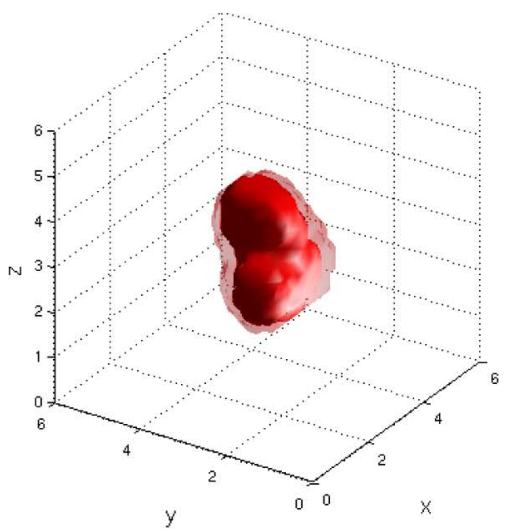

(a)

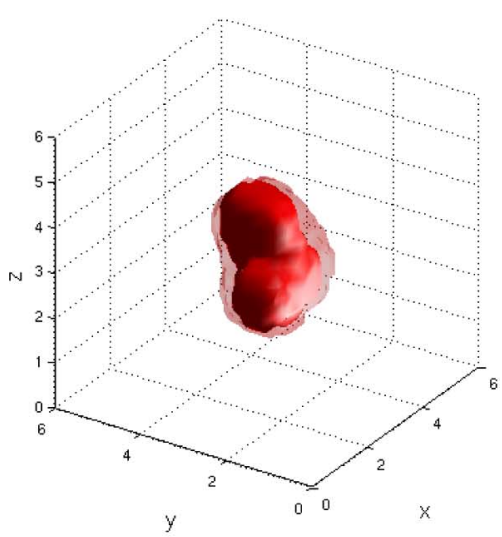

(b)

Fig. 7. Visualization of the shape uncertainty region for a third-order shape, with a phase noise variance of $1^{\circ}$. (a) Uncertainty in the case where the center and absorption contrast are known a priori. (b) Uncertainty in the case where the center and absorption contrast are not known a priori.

shape uncertainty, but to a relatively modest degree, less than $15 \%$.

We note that the confidence regions shown in Fig. 7 may be somewhat pessimistic, in that we are computing the maximum perturbation at each point of the boundary given that the shape parameters are within a $95 \%$ confidence interval. It may be that a method, such as that described in [47] may give a somewhat less conservative estimate of the shape confidence region.

\section{CONCLUSION AND FUtURE WORK}

In this paper, we have shown that highly accurate estimation of object boundaries is possible in DOT, an imaging modality with typically very low spatial resolution, even with realistic levels of noise added to the measurements. We have demonstrated a Newton-type image-reconstruction algorithm for the nonlinear reconstruction of absorbing inhomogeneities which are simply connected and polar in shape. With the shape boundaries represented using the real and imaginary parts of the spherical harmonic functions, our algorithm simulataneously estimates object shape, center position, and contrast, making use of constrained estimation with a centroid constraint to ensure the uniqueness of the shape/center representation. We are able to compute the sensitivity of the cost function to perturbations in object shape, center position, and contrast directly, by making use of shape derivative theory, thus obviating the need for computationally expensive finite-difference computations. Given knowledge of these sensitivities, and making use of the constrained and unconstrained Cramér-Rao lower bounds, we are able to compute a lower bound on the uncertainty in our estimates of shape parameters for a given absorbing object, source-detector configuration, and level of measurement noise. In addition to giving us a confidence region on the shape estimates which we obtain, this information can help us to design sensors and to determine the best-case performance of an imaging system with known characteristics.

We hope that this work will be of use in improving the resolution of DOT and in making the results more quantitatively useful, particularly in the estimation of the contrast and size of tumors in the breast. Future work will extend the results in this paper to the problem of simultaneously estimating an object's absorption and scattering contrast, as well as to the problem of reconstructing objects using measurements at multiple wavelengths, incorporating spectroscopic constraints.

\section{APPENDIX \\ DERIVATION OF (15)}

In what follows, we make use of a similar procedure to that used in [48]. Multiplying (12) by $u_{d}(\mathbf{r}, \omega)$ and integrating over $\Omega_{e}$ we find

$$
\begin{aligned}
& -\int_{\Omega_{e}}\left[D_{e} u_{d}(\mathbf{r}, \omega) \nabla^{2} u_{s}(\mathbf{r}, \omega)\right. \\
& \left.+\left(\mu_{a}^{e}-\frac{j \omega}{v}\right) u_{d}(\mathbf{r}, \omega) u_{s}(\mathbf{r}, \omega)\right] d \mathbf{r}=0 .
\end{aligned}
$$

Using the divergence theorem, this expression can be rewritten as

$$
\begin{aligned}
& -\int_{\Gamma_{e}} D_{e} u_{d}(\mathbf{s}, \omega) \frac{\partial u_{s}(\mathbf{s}, \omega)}{\partial \boldsymbol{\nu}} d \mathbf{s} \\
& \quad-\int_{\Gamma_{i}} D_{e} u_{d}(\mathbf{s}, \omega) \frac{\partial u_{s, e}(\mathbf{s}, \omega)}{\partial \boldsymbol{\nu}} d \mathbf{s} \\
& \quad+\int_{\Omega_{e}} D_{e} \nabla u_{s}(\mathbf{r}, \omega) \cdot \nabla u_{d}(\mathbf{r}, \omega) d \mathbf{r} \\
& \quad+\int_{\Omega_{e}}\left[\mu_{a}^{e}-\frac{j \omega}{v}\right] u_{s}(\mathbf{r}, \omega) u_{d}(\mathbf{r}, \omega) d \mathbf{r}=0 .
\end{aligned}
$$

Likewise, multiplying (12) by $u_{d}(\mathbf{r}, \omega)$, integrating over $\Omega_{i}$, and using the divergence theorem, we find

$$
\begin{aligned}
& \int_{\Gamma_{i}} D_{i} u_{d}(\mathbf{s}, \omega) \frac{\partial u_{s, i}(\mathbf{s}, \omega)}{\partial \boldsymbol{\nu}} d \mathbf{s} \\
& \quad+\int_{\Omega_{i}} D_{i} \nabla u_{s}(\mathbf{r}, \omega) \cdot \nabla u_{d}(\mathbf{r}, \omega) d \mathbf{r} \\
& \quad+\int_{\Omega_{i}}\left[\mu_{a}^{i}-\frac{j \omega}{v}\right] u_{s}(\mathbf{r}, \omega) u_{d}(\mathbf{r}, \omega) d \mathbf{r}=0 .
\end{aligned}
$$

Summing (40) and (41) and applying the boundary condition that $D_{i}\left(\partial u_{s, i}(\mathbf{s}, \omega) / \partial \boldsymbol{\nu}\right)=D_{e}\left(\partial u_{s, e}(\mathbf{s}, \omega) / \partial \boldsymbol{\nu}\right)$ on $\Gamma_{i}$, we find

$$
\begin{aligned}
& -\int_{\Gamma_{e}} D_{e} u_{d}(\mathbf{s}, \omega) \frac{\partial u_{s}(\mathbf{s}, \omega)}{\partial \boldsymbol{\nu}} d \mathbf{s} \\
& \quad+\int_{\Omega} D_{e} \nabla u_{s}(\mathbf{r}, \omega) \cdot \nabla u_{d}(\mathbf{r}, \omega) d \mathbf{r}
\end{aligned}
$$




$$
\begin{aligned}
& +\int_{\Omega_{i}} D \nabla u_{s}(\mathbf{r}, \omega) \cdot \nabla u_{d}(\mathbf{r}, \omega) d \mathbf{r} \\
& +\int_{\Omega}\left[\mu_{a}^{e}-\frac{j \omega}{v}\right] u_{s}(\mathbf{r}, \omega) u_{d}(\mathbf{r}, \omega) d \mathbf{r} \\
& +\int_{\Omega_{i}} \mu_{a} u_{s}(\mathbf{r}, \omega) u_{d}(\mathbf{r}, \omega) d \mathbf{r}=0
\end{aligned}
$$

where $\Omega=\Omega_{i} \cup \Omega_{e}$. Finally, from (12) we see that $\left(\partial u_{s}(\mathbf{r}, \omega) / \partial \boldsymbol{\nu}\right)=\left(1 / D_{e}\right) \delta\left(\mathbf{r}-\mathbf{r}_{s}\right)-\left(\beta / D_{e}\right) u_{s}(\mathbf{r}, \omega)$ on $\Gamma_{e}$. Using this result in (42), we obtain

$$
\begin{gathered}
-u_{d}\left(\mathbf{r}_{s}, \omega\right)+\int_{\Gamma_{e}} \beta u_{s}(\mathbf{s}, \omega) u_{d}(\mathbf{s}, \omega) d \mathbf{s} \\
+\int_{\Omega} D_{e} \nabla u_{s}(\mathbf{r}, \omega) \cdot \nabla u_{d}(\mathbf{r}, \omega) d \mathbf{r} \\
+\int_{\Omega_{i}} D \nabla u_{s}(\mathbf{r}, \omega) \cdot \nabla u_{d}(\mathbf{r}, \omega) d \mathbf{r} \\
+\int_{\Omega}\left[\mu_{a}^{e}-\frac{j \omega}{v}\right] u_{s}(\mathbf{r}, \omega) u_{d}(\mathbf{r}, \omega) d \mathbf{r} \\
+\int_{\Omega_{i}} \mu_{a} u_{s}(\mathbf{r}, \omega) u_{d}(\mathbf{r}, \omega) d \mathbf{r}=0 .
\end{gathered}
$$

Applying the same procedure, we multiply (14) by $u_{s}(\mathbf{r}, \omega)$ and integrate over $\Omega$

$$
\begin{aligned}
& \int_{\Omega}\left[-D_{e} u_{s}(\mathbf{r}, \omega)\right. \nabla^{2} u_{d}(\mathbf{r}, \omega) \\
&\left.+\left(\mu_{a}^{e}-\frac{j \omega}{v}\right) u_{s}(\mathbf{r}, \omega) u_{d}(\mathbf{r}, \omega)\right] d \mathbf{r}=0
\end{aligned}
$$

Using the divergence theorem, we find

$$
\begin{aligned}
& -\int_{\Gamma_{e}} D_{e} u_{s}(\mathbf{s}, \omega) \frac{\partial u_{d}(\mathbf{s}, \omega)}{\partial \boldsymbol{\nu}} d \mathbf{s} \\
& \quad+\int_{\Omega} D_{e} \nabla u_{s}(\mathbf{r}, \omega) \cdot \nabla u_{d}(\mathbf{r}, \omega) d \mathbf{r} \\
& \quad+\int_{\Omega}\left[\mu_{a}^{e}-\frac{j \omega}{v}\right] u_{s}(\mathbf{r}, \omega) u_{d}(\mathbf{r}, \omega) d \mathbf{r}=0 .
\end{aligned}
$$

Finally, we apply the boundary condition from (14) that $\left(\partial u_{d}(\mathbf{r}, \omega) / \partial \boldsymbol{\nu}\right)=\left(1 / D_{e}\right) \delta\left(\mathbf{r}-\mathbf{r}_{d}\right)-\left(\beta / D_{e}\right) u_{d}(\mathbf{r}, \omega)$ to $(45)$

$$
\begin{aligned}
& -u_{s}\left(\mathbf{r}_{d}, \omega\right)+\int_{\Gamma_{e}} \beta u_{s}(\mathbf{s}, \omega) u_{d}(\mathbf{s}, \omega) d \mathbf{s} \\
& +\int_{\Omega} D_{e} \nabla u_{s}(\mathbf{r}, \omega) \cdot \nabla u_{d}(\mathbf{r}, \omega) d \mathbf{r} \\
& +\int_{\Omega}\left[\mu_{a}^{e}-\frac{j \omega}{v}\right] u_{s}(\mathbf{r}, \omega) u_{d}(\mathbf{r}, \omega) d \mathbf{r}=0 .
\end{aligned}
$$

Subtracting (46) from (43), we obtain

$$
\begin{aligned}
& u_{s}\left(\mathbf{r}_{d}, \omega\right)-u_{d}\left(\mathbf{r}_{s}, \omega\right) \\
& \quad+\int_{\Omega_{i}} D \nabla u_{s}(\mathbf{r}, \omega) \cdot \nabla u_{d}(\mathbf{r}, \omega) d \mathbf{r} \\
& \quad+\int_{\Omega_{i}} \mu_{a} u_{s}(\mathbf{r}, \omega) u_{d}(\mathbf{r}, \omega) d \mathbf{r}=0
\end{aligned}
$$

from which (15) follows.

\section{ACKNOWLEDGMENT}

The authors would like to thank the anonymous reviewers for their careful reading of the manuscript and for offering a number of useful suggestions.

\section{REFERENCES}

[1] V. A. Markel and J. C. Schotland, "The inverse problem in optical diffusion tomography. I. Fourier-Laplace inversion formulas," J. Opt. Soc. Amer., vol. 18, no. 6, pp. 1336-1347, Jun. 2001.

[2] V. A. Markel, J. A. O'Sullivan, and J. C. Schotland, "Inverse problem in optical diffusion tomography. IV. Nonlinear inversion formulas," $J$. Opt. Soc. Amer. A, vol. 20, no. 5, pp. 903-912, 2003.

[3] B. W. Pogue, S. P. Poplack, T. O. McBride, W. A. Wells, K. S. Osterman, U. L. Osterberg, and K. D. Paulsen, "Quantitative hemoglobin tomography with diffuse near-infrared spectroscopy: Pilot results in the breast," Radiology, vol. 218, no. 1, pp. 261-266, 2001.

[4] A. E. Cerussi, A. J. Berger, F. Bevilacqua, N. Shah, D. Jakubowski, J. Butler, R. F. Holcombe, and B. J. Tromberg, "Sources of absorption and scattering contrast for near-infrared optical mammography," Acad. Radiol., vol. 8, no. 3, pp. 211-218, 2001.

[5] T. O. McBride, B. W. Pogue, E. D. Gerety, S. R. Poplack, U. L. Osterberg, and K. D. Paulsen, "Spectroscopic diffuse optical tomography for the quantitative assessment of hemoglobin concentration and oxygen saturation in breast tissue," Appl. Opt., vol. 38, no. 25, pp. 5480-5490, 1999.

[6] A. P. Gibson, J. C. Hebden, and S. R. Arridge, "Recent advances in diffuse optical imaging," Phys. Med. Biol., vol. 50, pp. R1-R43, Feb. 2005.

[7] H. Dehghani, B. W. Pogue, S. P. Poplack, and K. D. Paulsen, "Multiwavelength 3-D near-infrared tomography of the breast: Initial simulation, phantom, and clinical results," Appl. Opt., vol. 42, no. 1, pp. 135-145, 2003.

[8] S. Arridge and M. Schweiger, "A gradient-based optimisation scheme for optical tomography," Opt. Express, vol. 2, no. 6, pp. 213-226, 1998.

[9] P. K. Yalavarthy, B. W. Pogue, H. Dehghani, and K. D. Paulsen, "Weight-matrix structured regularization provides optimal generalized least-squares estimate in diffuse optical tomography," Med. Phys., vol. 24, pp. 2085-2098, Jun. 2007.

[10] S. R. Arridge, "Photon measurement density functions. Part 1: Analytical forms," Appl. Opt., vol. 34, no. 31, pp. 7395-7409, 1995.

[11] P. C. Hansen, Rank-Deficient and Discrete Ill-Posed Problems. Philadelphia, PA: SIAM, 1998.

[12] C. R. Vogel, Computational Methods For Inverse Problems. Philadelphia, PA: SIAM, 2002.

[13] H. Jiang, K. D. Paulsen, U. L. Osterberg, B. W. Pogue, and M. S. Patterson, "Optical image reconstruction using frequency domain data simulations and experiments," J. Opt. Soc. Amer. A., vol. 13, no. 2, pp. 253-266, 1996.

[14] K. D. Paulsen and H. Jiang, "Enhanced frequency-domain optical image reconstrution in tissues through total-variation minimization," Appl. Opt., vol. 35, no. 19, pp. 3447-3458, 1996.

[15] P. Charbonnier, L. Blanc-Feraud, G. Aubert, and M. Barlaud, "Deterministic edge-preserving regularization in computed imaging," IEEE Trans. Image Process., vol. 6, no. 2, pp. 298-311, Feb. 1997.

[16] C. R. Vogel and M. E. Oman, "Fast, robust total variation-based reconstruction of noisy, blurred images," IEEE Trans. Image Process., vol. 7, no. 7, pp. 813-824, Jul. 1998.

[17] Y. Li and F. Santosa, "A computational algorithm for minimizing total variation in image reconstruction," IEEE Trans. Image Process., vol. 5, no. 6, pp. 987-995, Jun. 1996.

[18] D. Freedman, R. Radke, Y. Jeong, T. Zhang, D. M. Lovelock, and G. T. Y. Chen, "Model-based segmentation of medical imagery by matching distributions," IEEE Trans. Med. Imag., vol. 24, no. 3, pp. 281-292, Mar. 2005.

[19] J. Li, "Three dimensional shape modeling: Segmentation, reconstruction and reconstruction," Ph.D. dissertation, Univ. Michigan, Ann Arbor, MI, 2002.

[20] B. Brooksby, B. W. Pogue, S. Jiang, H. Dehghani, S. Srinivasan, C. Kogel, T. D. Tosteson, J. Weaver, S. P. Poplack, and K. D. Paulsen, "Imaging breast adipose and fibroglandular tissue molecular signatures by using hybrid MRI-guided near-infrared spectral tomography.," Proc. Natl. Acad. Sci. USA, vol. 103, pp. 8828-8833, Jun. 2006.

[21] F. Santosa, "A level-set approach for inverse problems involving obstacles," ESAIM: Control, Optimization, Calculus Variations, vol. 1, pp. 17-33, 1996.

[22] H. Feng, W. C. Karl, and D. A. Casta\&amp;ntilde;on, "A curve evolution approach to object-based tomographic reconstruction," IEEE Trans. Image Process., vol. 12, no. 1, pp. 44-57, Jan. 2003.

[23] J. C. Ye, Y. Bresler, and P. Moulin, "Cramer-Rao bounds for 2-D target shape estimation in nonlinear inverse scattering problems with application to passive radar," IEEE Trans. Ant. Propogat., vol. 49, no. 5, pp. 771-783, May 2001. 
[24] V. Kolehmainen, M. Vaukohnen, J. P. Kaipio, and S. R. Arridge, "Recovery of piecewise constant coefficients in optical diffusion tomography," Opt. Express, vol. 7, no. 13, pp. 468-480, 2000.

[25] A. O. Hero, R. Piramuthu, J. A. Fessler, and S. R. Titus, "Minimax emission computed tomography using high-resolution anatomical information and B-spline models," IEEE Trans. Inf. Theory, vol. 45, no. 3, pp. 920-938, Apr. 1999.

[26] M. E. Kilmer, E. L. Miller, D. A. Boas, and D. Brooks, "A shape-based reconstruction technique for DPDW data, invited paper," Opt. Express, vol. 7, no. 13, pp. 481-491, Dec. 2000.

[27] S. Babaeizadeh and D. H. Brooks, "Electrical impedance tomography for piecewise constant domains using boundary element shape-based inverse solutions," IEEE Trans. Med. Imag., vol. 26, no. 5, pp. 637-648, May 2007.

[28] A. D. Zacharopoulos, "3-D shape-based reconstructions in medical imaging," Ph.D. dissertation, Univ. London, London, U.K., 2005.

[29] J. Sokolowski and J. P. Zolesio, Introduction to Shape Optimization: Shape Sensitivity Analysis. Berlin, Germany: Springer-Verlag, 1992.

[30] A. Kirsch, "The domain derivative and two applications in inverse scattering theory," Inverse Problems, vol. 9, no. 1, pp. 81-96, 1993.

[31] F. Hettlich, "Frechet derivatives in inverse obstacle scattering," Inverse Problems, vol. 11, pp. 371-382, 1995.

[32] R. Potthast, "Frechet differentiability of boundary integral operators," Inverse Problems, vol. 10, pp. 431-447, 1994.

[33] T. Hohage and C. Schormann, "A newton-type method for a transmission problem in inverse scattering," Inverse Problems, vol. 14, pp. 1207-1227, 1998.

[34] J. C. Ye, Y. Bresler, and P. Moulin, "Asymptotic global confidence regions in parametric shape estimation problems," IEEE Trans. Inf. Theory, vol. 46, no. 5, pp. 1881-1895, Aug. 2000.

[35] P. Stoica and B. C. Ng, "On the cramer-rao bound under parametric constraints," IEEE Signal Process. Lett., vol. 5, pp. 177-179, Jul. 1998.

[36] P. Dennery and A. Krzywicki, Mathematics For Physicists. Mineola, NY: Dover, 1995

[37] S. R. Arridge, "Optical tomography in medical imaging," Inverse Problems, vol. 15, pp. R41-R93, 1999.
[38] R. C. Haskell, L. O. Svaasand, T. Tsay, T. Feng, M. S. McAdams, and B. J. Tromberg, "Boundary conditions for the diffusion equation in radiative transfer," J. Opt. Soc. Amer., vol. 11, pp. 2727-2741, 1994.

[39] D. L. Luenberger, Optimization by Vector Space Methods. New York: Wiley, 1969.

[40] J. Nocedal and S. J. Wright, Numerical Optimization. New York: Springer, 2006.

[41] A. O. Hero, J. A. Fessler, and M. Usman, "Exploring estimator biasvariance tradeoffs using the uniform CR bound," IEEE Trans. Signal Process., vol. 44, no. 8, pp. 2026-2041, Aug. 1996.

[42] R. D. Gill and B. Y. Levitt, "Applications of the van trees inequality: A Bayesian Cramér-Rao bound," Bernoulli, vol. 1, no. 1/2, pp. 59-79, Mar. 1995.

[43] Y. Saad and M. H. Schultz, SIAM J. Sci. Statist. Comput. "GMRES: A generalized minimal residual algorithm for solving nonsymmetric linear systems," 1986, vol. 7, pp. 856-869.

[44] P. C. Hansen, "Analysis of discrete ill-posed problems by means of the 1-curve," SIAM Rev., vol. 34, no. 4, pp. 561-580, Dec. 1992.

[45] P. C. Hansen and D. P. O'Leary, "The use of the l-curve in the regularization of discrete ill-posed problems," SIAM J. Sci. Comput., vol. 14, pp. 1487-1503, 1993.

[46] D. A. Boas, M. A. O'Leary, and B. Chance, "Scattering of diffuse photon density waves by spherical inhomogeneities within turbid media: Analytical solution and applications," Proc. Nat. Acad. Sci. USA, vol. 91, pp. 4887-4891, 1994.

[47] J. C. Ye, P. Moulin, and Y. Bresler, "Asymptotic global confidence regions for 3D parametric shape estimation in inverse problems," IEEE Trans. Image Process., vol. 15, no. 10, pp. 2904-2919, Oct. 2006.

[48] A. D. Zacharopoulos, S. R. Arridge, O. Dorn, V. Kolehmainen, and J. Sikora, "3-D reconstruction of shape and piecewise constant region values for optical tomography using spherical harmonic parametrization and a boundary element method," Inverse Problems, vol. 22, pp. 1509-1532, 2006. 